\title{
An independent SSVEP-based brain-computer interface in locked-in syndrome
}

\author{
D Lesenfants ${ }^{1}$, D Habbal ${ }^{1}$, Z Lugo $^{1,3}$, M Lebeau $^{1}$, P Horki $^{2}$, E Amico $^{1}$, \\ C Pokorny ${ }^{2}$, F Gómez ${ }^{1,4}$, A Soddu ${ }^{1,5}$, G Müller-Putz ${ }^{2}$, S Laureys ${ }^{1,6}$ \\ and $Q$ Noirhomme ${ }^{1,6}$ \\ ${ }^{1}$ Coma Science Group, Cyclotron Research Centre and Neurology department, University of Liège \\ Liège, Belgium \\ ${ }^{2}$ Laboratory of Brain-Computer Interfaces, Institute for Knowledge Discovery, Graz University of \\ Technology, Graz, Austria \\ ${ }^{3}$ Department of Psychology I, Institute for Psychology, University of Würzburg, Würzburg, Germany \\ ${ }^{4}$ Combios Laboratory, Computer Science Department, Universidad Central, Bogotá, Colombia \\ ${ }^{5}$ Brain \& Mind Institute, Physics \& Astronomy Department, University of Western Ontario, London \\ ON, Canada \\ E-mail: Damien.Lesenfants@doct.ulg.ac.be
}

Received 9 January 2014, revised 18 March 2014

Accepted for publication 21 March 2014

Published 19 May 2014

\begin{abstract}
Objective. Steady-state visually evoked potential (SSVEP)-based brain-computer interfaces (BCIs) allow healthy subjects to communicate. However, their dependence on gaze control prevents their use with severely disabled patients. Gaze-independent SSVEP-BCIs have been designed but have shown a drop in accuracy and have not been tested in brain-injured patients. In the present paper, we propose a novel independent SSVEP-BCI based on covert attention with an improved classification rate. We study the influence of feature extraction algorithms and the number of harmonics. Finally, we test online communication on healthy volunteers and patients with locked-in syndrome (LIS). Approach. Twenty-four healthy subjects and six LIS patients participated in this study. An independent covert two-class SSVEP paradigm was used with a newly developed portable light emitting diode-based 'interlaced squares' stimulation pattern. Main results. Mean offline and online accuracies on healthy subjects were respectively $85 \pm 2 \%$ and $74 \pm 13 \%$, with eight out of twelve subjects succeeding to communicate efficiently with $80 \pm 9 \%$ accuracy. Two out of six LIS patients reached an offline accuracy above the chance level, illustrating a response to a command. One out of four LIS patients could communicate online. Significance. We have demonstrated the feasibility of online communication with a covert SSVEP paradigm that is truly independent of all neuromuscular functions. The potential clinical use of the presented BCI system as a diagnostic (i.e., detecting command-following) and communication tool for severely brain-injured patients will need to be further explored.
\end{abstract}

Keywords: SSVEP-BCI, locked-in Syndrome, gaze-independent, feature extraction, harmonic

\section{Introduction}

Brain-computer interfaces (BCIs) [1] translate measures of brain activity into messages or commands and provide a direct

${ }^{6}$ Both authors contributed equally. connection between the human brain and a computer. The most favorable noninvasive brain imaging method employed in BCI is electroencephalography (EEG), in which electrical signals of high temporal resolution are recorded from the scalp. The existing EEG-based BCI designs rely on a variety of different EEG signal features, for example slow cortical 
potentials [2], mu rhythms [3], P300 potentials [4] and steadystate visually evoked potentials (SSVEPs) $[5,6]$. In SSVEPbased BCIs, one or more stimuli oscillating at different constant frequencies are presented to the subject. When the subject focuses his attention on the stimulus, EEG activity is detected at the corresponding frequency over occipital areas [7]. The SSVEP-based BCI has many advantages over other EEG-based BCI systems, including (i) a high signal-tonoise ratio, (ii) a high information transfer rate [8], (iii) less susceptibility to eye movements and blink artifacts [9] as well as to electromyographic artifacts [10], and (iv) they require very little training since the SSVEP is an inherent response of the brain.

BCIs have been proposed as a diagnostic tool for the detection of consciousness and/or a communication tool for severely brain-injured patients, and especially patients with locked-in syndrome (LIS) [11, 12]. Following a brainstem lesion, these patients often remain comatose for some days or weeks, needing artificial respiration, and then gradually wake up but remain paralyzed and voiceless. In acute LIS, the difficulty to recognize unambiguous signs of consciousness, the extreme motor disabilities, the apparent similarity with the vegetative state/unresponsive wakefulness syndrome (i.e., eyes opening and motor immobility without signs of awareness) [13] and the fluctuations of arousal levels [14] often result in the diagnosis being delayed or even missed [15]. In the chronic stage, computer-based communication could improve a patient's quality of life and increase interaction with their environment.

Current BCIs relying on VEPs depend on gaze control $[16,17]$ and thus fall into the category of dependent BCIs [1]. Therefore, these BCIs are not applicable to those whose severe disabilities extend to impaired or nonexistent ocular motor control, such as LIS patients in which (i) eye movements may be inconsistent, very small and easily exhausted in the acute stage and (ii) deteriorated or nonexistent oculomotor control could be observed in the chronic stage. Independent SSVEPBCIs based on covert attention have been proposed [18-20] but have shown a drop in robustness in healthy subjects and have never been tested on patients.

The SSVEPs have the same fundamental frequency (first harmonic) as the stimulating frequency, but usually they also include higher [21] and/or sub-harmonic frequencies [22]. Previous SSVEP-based BCIs were implemented on the basis of the first [7] or on the first and second harmonic detection $[5,6,23]$. Recent research studied the impact of harmonic frequency components in the classification accuracy and showed that the use of higher harmonics positively influence classification in overt SSVEP [16]. The influence of harmonics in covert SSVEP has never been studied.

The aim of the present work was to develop a novel covert SSVEP-BCI with an improved classification rate enabling functional communication. To achieve this goal, we have: (i) proposed a new portable covert stimulation pattern enabling a better discrimination between two stimuli, (ii) tested different feature extraction algorithms, (iii) studied the influence of the number of harmonics (which has never been tested in covert SSVEP), and (iv) developed and tested on 12 healthy subjects an online covert SSVEP-BCI which allows synchronous communication without ocular motor control. The potential use of the system as an offline diagnostic tool and/or online communication system for the disabled was then assessed in six patients with LIS.

\section{Materials and methods}

\subsection{Subjects}

Twelve healthy subjects (five men; age range $22-43$ years; mean $\pm \mathrm{SD}: 28.2 \pm 5.7$, hereinafter called group A, participated in the offline study. Analysis on group A was used to determine the best feature extraction algorithm and to study the influence of parameters (i.e. the automatic channel selection algorithm (ACSA), the number of harmonics and the classifiers). Then, the parameters defined from group A were applied online to a second group, hereinafter called group $\mathrm{B}$, composed of 12 different healthy subjects (two men; age range $21-30$ years; $24.1 \pm 3.0$ ) and six LIS patients (four men; age range 23-74 years; $49.0 \pm 19.7$; see table 3 ). None had prior experience with BCI. The study was approved by the ethical committee of the University Hospital of Liège and all participants or their legal representatives provided informed consent.

\subsection{Data collection}

EEG signals were recorded from $12 \mathrm{Ag} / \mathrm{AgCl}$ ring electrodes at locations $P_{3}, P_{1}, P_{2}, P_{4}, P_{7}, P_{3}, P_{z}, P_{4}, P_{8}, O_{1}$, $O_{z}$ and $O_{2}$, referenced to $P_{z}$, based on the international 10-20 electrode system. A ground electrode was placed behind the right mastoid. All impedances were kept below $5 \mathrm{k} \Omega$. Eye movements were monitored with four electrodes: two on the left and right temples; the remaining two over and under the supra-orbital ridge respectively. The electroencephalograms were recorded using a BrainVision V-Amp amplifier with a band pass filter set between 0.01 and $100 \mathrm{~Hz}$ and a sampling frequency of $250 \mathrm{~Hz}$.

\subsection{Paradigms}

2.3.1. Group A. The visual stimulation was delivered via a custom made stimulation unit, which can be decomposed into a control unit and a stimulation panel, based on the paradigm introduced in [24]. The panel, placed at $30 \mathrm{~cm}$ from subject's head, is a $7 \times 7 \mathrm{~cm}^{2}$ 'interlaced square' made of red and yellow $1 \times 1 \mathrm{~cm}^{2}$ light emitting diode (LED)squares with a white fixation cross in the middle (see figure 1). The interlaced square pattern showed a $10 \%$ improvement in accuracy in comparison with a 'line' pattern [19]. The control unit is an electronic embedded system used for precise control of red and yellow flickering frequencies, which can be varied independently between 1 and $99 \mathrm{~Hz}$ by a programmable integrated circuit microcontroller. During the experiments, the yellow and red squares were programmed to flicker at $10 \mathrm{~Hz}$ and $14 \mathrm{~Hz}$ respectively (duty cycle $=0.5$ ). This stimulation system has the advantages of being small, portable and easy to use at the patient's bedside. Each subject underwent a total 


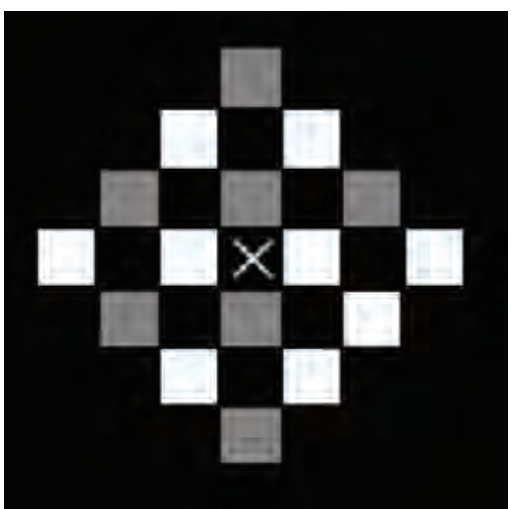

Figure 1. Electronic visual stimulation unit. The yellow squares (represented by white squares here) flicker at the frequency of $10 \mathrm{~Hz}$. The red squares (represented by gray squares here) flash at $14 \mathrm{~Hz}$.

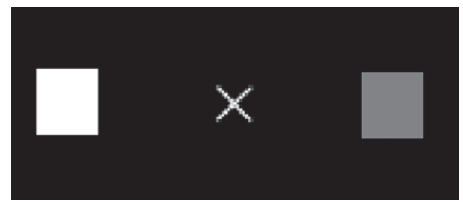

Figure 2. Overt block pattern. The yellow square (represented by the white square) flashes at $10 \mathrm{~Hz}$. The red square (represented by the gray square) flashes at $14 \mathrm{~Hz}$.

of six runs, each lasting about $5 \mathrm{~min}$. Each run contained ten $7 \mathrm{~s}$ trials [24], separated by $23 \mathrm{~s}$ periods ( $7 \mathrm{~s}$ of rest and $16 \mathrm{~s}$ of auditory instructions delivered via headphones). During a run, the interlaced squares pattern was continuously flashing and an equal number of both stimuli was presented in a random order. The subject was instructed to fix his/her gaze on the white cross in the middle and to focus attention on one of the flashing colors. The inter-run rest periods were left at the discretion of the subject and lasted between 2 to $10 \mathrm{~min}$.

2.3.2. Group B. Previous to the training session, three healthy subjects and four LIS patients from group B performed an overt session. The subjects were seated about $30 \mathrm{~cm}$ from a block pattern (see figure 2), containing a yellow and a red stimulus flashing at $f_{1}=10 \mathrm{~Hz}$ and $f_{2}=14 \mathrm{~Hz}$ (duty cycle $=0.5$ ) respectively. This pattern was composed of two $2 \times 2$ $\mathrm{cm}^{2}$ blocks made of $1 \times 1 \mathrm{~cm}^{2}$ LED squares separated by $12 \mathrm{~cm}$ with a white fixation cross in between. Then, the subjects had a training session identical to group A. After a short break to train the classifier, they performed an online communication session. Thirty-three yes/no questions were asked synchronously to the subject (e.g. 'is your name Paul ?', 'are you 25 years old ?'). Answers needed to be unambiguous and were known a priori. The subjects had to focus their attention over $7 \mathrm{~s}$ on the yellow flashes to answer 'yes' or on the red for 'no'. The stimulation panel was activated during the question/response time only to avoid tiredness.

\subsection{Data analysis}

EEG signals were preprocessed with a Butterworth fourthorder low-pass filter with a cutoff frequency of $60 \mathrm{~Hz}$ and a Butterworth fourth-order high-pass filter with a cutoff frequency of $5 \mathrm{~Hz}$. An IIR notch filter ( $\mathrm{fc}=50 \mathrm{~Hz}, \mathrm{Q}=$ 35) was also applied to the data. Epochs of $7 \mathrm{~s}$ were used as a unique window.

For group A, frequency features were extracted from each epoch with four state-of-the-art feature extraction algorithms proposed in the literature: (1) discrete-time Fourier transform (DFT), (2) multitapers spectral analysis (PMTM) [25, 26], (3) canonical correlation analysis (CCA) [8] and (4) lockin analyzer system (LAS) [16, 17, 27]. The first, second and third harmonics of each stimulation frequency were extracted. Several feature sets were tested with one, two or all harmonics. An ACSA based on distinction sensitive learning vector quantization (DSLVQ) [28] selected an optimal channel set specific to each subject. Inside the classification process, this algorithm first computed the relevance of the monopolar input channels for all points in time during the course of a trial. If the mean classification accuracy inside the DSLVQ system was greater than random [29], the time series of relevance values were scaled and combined into one single relevance value for each channel. If this mean accuracy was at chance level, a subset of channels could not be extracted from the complete set of channels. Finally, channels with the highest relevance were automatically selected and features associated to these channel subsets were extracted for classification. Classification performances were computed with a linear discriminant analysis (LDA) or a linear support vector machine (SVM, linear kernel), and assessed with a $10 \times 10$ fold cross validation. A SVM classifier was used to study the influence of the number of harmonics. Note that increasing the number of features to process with a constant number of training trials prevented the use of LDA with three harmonics. The significance of the change in classification accuracy with the different approaches was assessed with a paired permutation test (results were considered significant at $p<0.05$ ) [30].

Optimal parameters defined offline in group A were applied online in group B. For group B, the classifier was trained on features extracted with PMTM on the first harmonic at channels selected by ACSA. A real-time auditory feedback 'the response to your question is YES/NO' was presented to the subject after each question.

Mean power spectra from $O_{2}$ during the $10 \mathrm{~Hz} 7 \mathrm{~s}$ trials were extracted offline with multitaper spectral analysis to illustrate typical SSVEP responses for healthy subjects and LIS patients during overt and covert conditions (see figure 3). All analyses were done with custom-made codes using Matlab and Graz DSLVQ toolbox (Laboratory of Brain-Computer Interfaces, Institute for Knowledge Discovery, Graz University of Technology, Austria). BCI2000 software package [31] and Fieldtrip Toolbox [32] were used for data acquisition and presentation of the auditory instructions. 

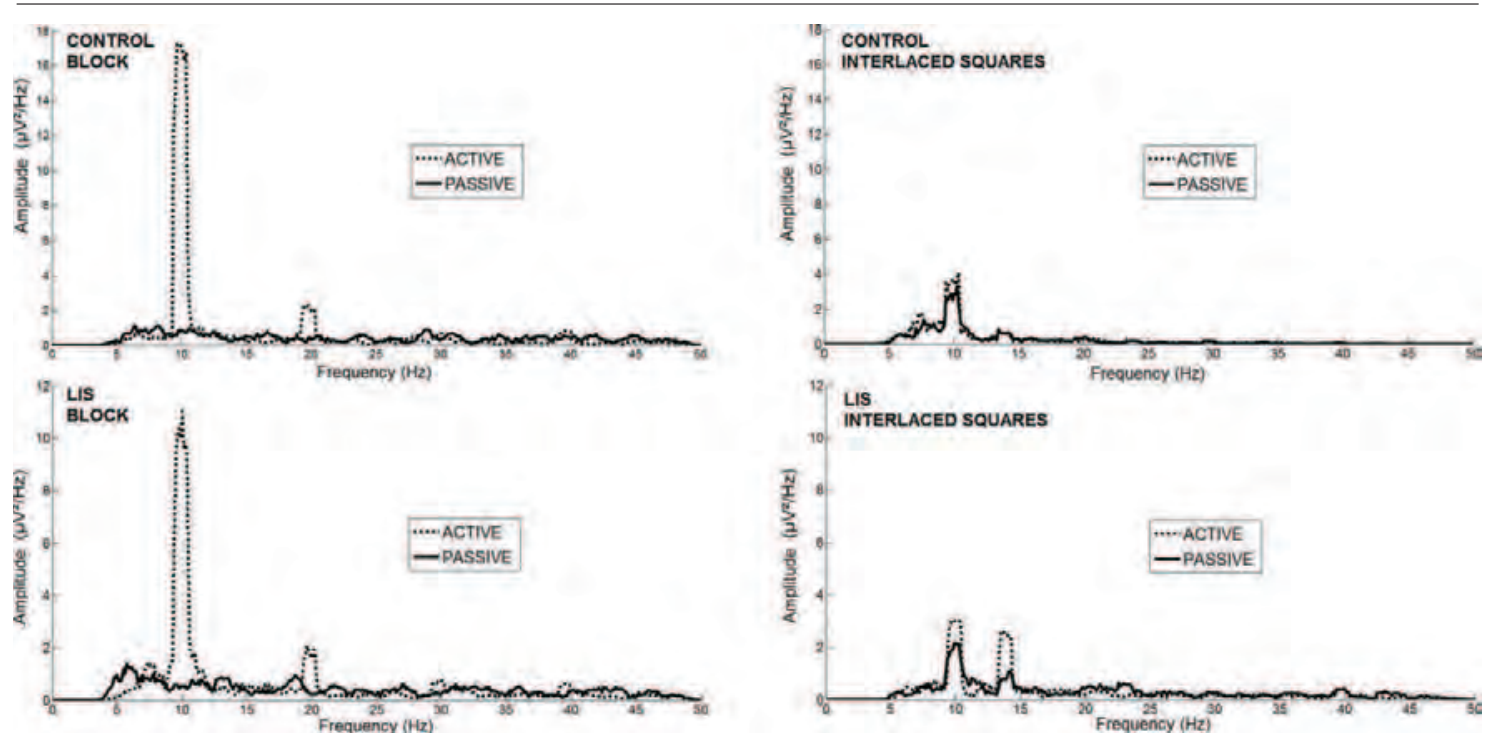

Figure 3. Mean power spectra, estimated with multitaper spectral analysis, recorded in a healthy subject (upper) and a patient with locked-in syndrome (lower) from electrode $\mathrm{O}_{2}$ during an 'overt' run using a block pattern (left) and a 'covert' run using an 'interlaced squares' pattern (right). Power spectrum obtained when the subject passively looked at the pattern (full line) and when the subject actively focussed attention on the target stimulus (dotted line). Note the attentional modulation in the control subject and the LIS patient in the two conditions (block and interlaced squares)

\section{Results}

Assessment of the electrooculogram did not show any eye movement during the covert trials for both groups. A binomial test [33] evaluated the chance level at $63 \%(\alpha=0.05,60$ trials). An online correct response rate (CRR) of $70 \%$ was considered the lowest rate of performance necessary to achieve efficient communication in a BCI with binary choice [37].

\subsection{Overt versus covert conditions}

Figure 3 (upper) shows typical overt and covert power spectra for a healthy subject. When a subject overtly focused on a stimulus, the power at the stimulation frequency and its second harmonic were clearly increased (see figure 3 , left). In the 'covert' condition (see figure 3, right), the power at the target's harmonic was smaller and could not be differentiated from surrounding frequencies. A peak at the non-target frequency could be observed with an amplitude close to the target amplitude. The same frequency behavior could be observed in patients with LIS (see figure 3, lower).

3.2. The influence of frequency feature extraction algorithm, automatic channel selection algorithm, number of harmonics and classifiers

The impact of the feature extraction algorithms was evaluated in ten out of 12 subjects from group A. Subjects SC11A and $\mathrm{SC} 12 \mathrm{~A}$ were rejected as all analyses showed classification accuracies at chance level (see tables 1 and 2). Significant differences were assessed with a two-tailed permutation test (1000 permutations).
First, we compared the results obtained by the feature extraction methods using all channels and a single harmonic. PMTM obtained the maximum accuracy of $77.0 \pm 3.4 \%$ averaged among subjects, while LAS produced a not significantly different mean accuracy of $74.4 \pm 3.2 \%$ (see tables 1 and 2). DFT and CCA gave significantly worse results than PMTM and LAS with respectively $69.4 \pm 3.4 \%$ and $58.4 \pm 3.9 \%$.

Second, we compared the results obtained by the feature extraction methods using the ACSA and a single harmonic. PMTM and LAS produced significantly greater accuracy than DFT and CCA, with an accuracy of $84.7 \pm 2.0 \%$ and $83.1 \pm$ $2.3 \%$ respectively. DFT obtained a $79.3 \pm 2.7 \%$ accuracy. CCA reached $72.4 \pm 1.6 \%$ but in only five out of the ten subjects (the electrodes subset could not be extracted in the five remaining healthy subjects). The performance with and without ACSA could therefore not be compared with CCA. Using the ACSA significantly increased the accuracy with PMTM, LAS and DFT. For a single harmonic, we obtained a significant mean accuracy increase of 7.8\% for PMTM, 7.9\% for LAS and 7.6\% for DFT (see figure 4).

Studying the influence of the number of harmonics (Nharm), LDA showed a decrease of accuracy when tested on all electrodes, and a stable accuracy with ACSA (maximal deviation of $2.4 \%$ ). LDA without ACSA could not compute the classification accuracy with three harmonics. With an SVM classifier, adding the second and the third harmonics resulted in no significant difference (two-tailed permutation test, 1000 permutations) and classification accuracies were similar to those previously described with LDA. Then, we analyzed the results obtained with the second and the third harmonics alone (i.e. without including preceding harmonics). Results showed 
Table 1. Mean and standard deviation of classification accuracy (in percent) obtained with the Thomson multitaper method (PMTM) for different numbers of harmonics with (ACSA) and without (AC) the use of automatic channel selection algorithm.

\begin{tabular}{|c|c|c|c|c|c|c|}
\hline \multirow[b]{2}{*}{ Subject } & \multicolumn{2}{|c|}{ Nharm $=1$} & \multicolumn{2}{|c|}{ Nharm $=2$} & \multicolumn{2}{|c|}{ Nharm $=3$} \\
\hline & $\mathrm{AC}$ & ACSA & $\mathrm{AC}$ & ACSA & $\mathrm{AC}$ & ACSA \\
\hline $\mathrm{SC} 1 \mathrm{~A}$ & $78.4 \pm 3.4$ & $85.7 \pm 1.8$ & $73.9 \pm 4.7$ & $94.3 \pm 1.8$ & I & $94.4 \pm 2.2$ \\
\hline $\mathrm{SC} 2 \mathrm{~A}$ & $92.3 \pm 2.4$ & $94.8 \pm 1.0$ & $76.5 \pm 4.9$ & $91.3 \pm 1.6$ & l & $92.6 \pm 1.1$ \\
\hline SC3A & $78.4 \pm 3.4$ & $84.4 \pm 2.1$ & $73.9 \pm 4.7$ & $84.9 \pm 2.6$ & I & $80.6 \pm 3.0$ \\
\hline SC4A & $67.8 \pm 3.5$ & $76.0 \pm 2.3$ & $51.7 \pm 4.7$ & $76.7 \pm 3.7$ & l & $78.6 \pm 3.2$ \\
\hline SC5A & $62.4 \pm 3.9$ & $78.9 \pm 2.3$ & $51.6 \pm 4.8$ & $77.4 \pm 2.4$ & I & $70.5 \pm 3.2$ \\
\hline SC6A & $71.6 \pm 3.8$ & $85.8 \pm 2.6$ & $62.6 \pm 5.0$ & $81.9 \pm 3.3$ & 1 & $85.6 \pm 2.9$ \\
\hline SC7A & $89.5 \pm 2.4$ & $94.5 \pm 1.4$ & $76.0 \pm 3.9$ & $92.8 \pm 2.4$ & 1 & $92.6 \pm 1.9$ \\
\hline SC8A & $82.7 \pm 3.3$ & $89.2 \pm 2.3$ & $77.3 \pm 4.3$ & $94.4 \pm$ & I & $87.2 \pm 1.7$ \\
\hline SC9A & $91.1 \pm 2.3$ & $94.6 \pm$ & $84.0 \pm 4.3$ & $91.7 \pm$ & I & $91.8 \pm 1.3$ \\
\hline SC10A & $56.6 \pm 4.4$ & $63.5 \pm 1.8$ & $58.3 \pm 4.7$ & $67.5 \pm 3.0$ & I & $68.4 \pm 2.4$ \\
\hline SC11A & $57.0 \pm 3.8$ & I & $43.1 \pm 5.0$ & 1 & I & / \\
\hline $\mathrm{SC} 12 \mathrm{~A}$ & $44.5 \pm 3.8$ & I & $43.2 \pm 5.8$ & I & I & I \\
\hline Total & $77.0 \pm 3.4$ & $84.7 \pm 2.0$ & $68.6 \pm 4.6$ & $85.3 \pm 2.5$ & I & $84.2 \pm 2.4$ \\
\hline
\end{tabular}

Table 2. Mean and standard deviation of classification accuracy (in per cent) obtained with the lock-in analyzer system (LAS) for different numbers of harmonics with (ACSA) and without (AC) the use of automatic channel selection algorithm.

\begin{tabular}{|c|c|c|c|c|c|c|}
\hline \multirow[b]{2}{*}{ Subject } & \multicolumn{2}{|c|}{ Nharm $=1$} & \multicolumn{2}{|c|}{ Nharm $=2$} & \multicolumn{2}{|c|}{ Nharm $=3$} \\
\hline & $\mathrm{AC}$ & ACSA & $\mathrm{AC}$ & ACSA & $\mathrm{AC}$ & ACSA \\
\hline SC1A & $76.7 \pm 3.1$ & $85.0 \pm 1.9$ & $73.2 \pm 4.9$ & $93.9 \pm 1.8$ & I & $94.7 \pm 1.2$ \\
\hline SC2A & $86.5 \pm 2.1$ & $93.5 \pm 0.8$ & $4.8 \pm 5.2$ & $95.4 \pm 0.9$ & I & $95.0 \pm 1.7$ \\
\hline SC3A & $76.7 \pm 3.1$ & $82.5 \pm 2.7$ & $73.2 \pm 4.9$ & $79.4 \pm 2.7$ & I & $74.6 \pm 2.6$ \\
\hline SC4A & $61.0 \pm 4.2$ & $73.6 \pm 3.5$ & $58.3 \pm 4.5$ & $73.6 \pm 2.2$ & I & $77.6 \pm 1.9$ \\
\hline SC5A & $69.7 \pm 2.9$ & $80.2 \pm 2.4$ & $54.2 \pm 4.6$ & $76.1 \pm$ & I & $72.8 \pm 1.9$ \\
\hline SC6A & $61.4 \pm 3.6$ & $76.2 \pm 2.0$ & $60.8 \pm 4.7$ & $79.0 \pm 2.2$ & I & $77.7 \pm 3.3$ \\
\hline SC7A & $85.2 \pm 2.8$ & $90.0 \pm 2.2$ & $76.5 \pm 4.7$ & $91.2 \pm 2$ & l & $94.0 \pm 2.4$ \\
\hline SC8A & $83.0 \pm 2.9$ & $87.4 \pm 2$ & $75.9 \pm 4.4$ & $94.3 \pm$ & l & $91.5 \pm 2.6$ \\
\hline SC9A & $90.5 \pm 2.4$ & $92.9 \pm$ & $75.9 \pm 4.2$ & $90.9 \pm$ & I & $91.5 \pm 1.8$ \\
\hline SC10A & $53.7 \pm 3.8$ & $70.1 \pm 2.4$ & $61.7 \pm 4.7$ & $70.9 \pm 3.0$ & I & $71.4 \pm 2.9$ \\
\hline SC11A & $57.8 \pm 4.3$ & I & $48.5 \pm 5.1$ & I & I & / \\
\hline SC12A & $49.7 \pm 4.3$ & I & $54.0 \pm 5.0$ & I & I & I \\
\hline Total & $74.4 \pm 3.2$ & $83.1 \pm 2.8$ & $68.4 \pm 4.7$ & $84.5 \pm 2.3$ & I & $84.1 \pm 2.3$ \\
\hline
\end{tabular}

Table 3. Demographic and clinical data of patients with locked-in syndrome.

\begin{tabular}{|c|c|c|c|c|c|c|}
\hline & Gender & Age & Etiology & Interval (years) & MRI & Communication code \\
\hline LIS1 & M & 23 & $\begin{array}{l}\text { Traumatic } \\
\text { brainstem } \\
\text { lesion }\end{array}$ & & $\begin{array}{l}\text { Right cerebellar, right frontal and left } \\
\text { lenticular lesions. Diffuse axonal injury in } \\
\text { frontal and parietal lobes and the lenticular } \\
\text { capsula. Global cerebral atrophy with } \\
\text { quadriventriculaire hydrocephalus. }\end{array}$ & $\begin{array}{l}\text { Yes (looks right) and no } \\
\text { no (eyes closure) } \\
\text { communication. }\end{array}$ \\
\hline LIS2 & $\mathrm{F}$ & 56 & $\begin{array}{l}\text { Brainstem } \\
\text { stroke }\end{array}$ & 15 & $\begin{array}{l}\text { Ponto-mesencephalic, middle cerebellar and } \\
\text { occipital lesions. }\end{array}$ & $\begin{array}{l}\text { Yes-no head movements } \\
\text { communication } \\
\text { (nystagmus). }\end{array}$ \\
\hline LIS3 & M & 64 & $\begin{array}{l}\text { Brainstem } \\
\text { stroke }\end{array}$ & 12 & Pontine/diffuse peri-ventricular lesions. & $\begin{array}{l}\text { Yes-no head } \\
\text { movements } \\
\text { communication. }\end{array}$ \\
\hline LIS4 & $\mathrm{F}$ & 30 & Stroke & 9 & Cerebellar and brainstem lesions. & $\begin{array}{l}\text { Yes (eyes closure) and } \\
\text { no (looks up) communication } \\
\text { (nystagmus). }\end{array}$ \\
\hline LIS5 & M & 47 & $\begin{array}{l}\text { Brainstem } \\
\text { stroke }\end{array}$ & 3 & Cerebellar and ponto-mesencephalic lesions. & $\begin{array}{l}\text { Verbalization via } \\
\text { tracheostomy. }\end{array}$ \\
\hline LIS6 & M & 74 & $\begin{array}{l}\text { Brainstem } \\
\text { stroke }\end{array}$ & 3 & Ponto-mesencephalic and occipital lesions. & $\begin{array}{l}\text { Yes (head movement) and } \\
\text { no (eyes closure) } \\
\text { communication. }\end{array}$ \\
\hline
\end{tabular}




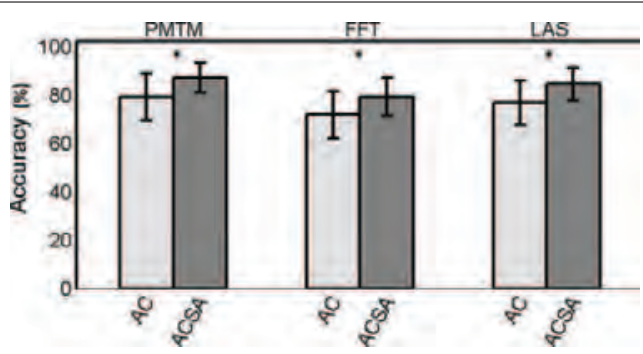

Figure 4. Recognition accuracies on 10 healthy subjects for different feature extraction algorithms and all channels (AC, white) or automatic channel selection algorithm (ACSA, gray).

a global decrease in accuracies when using the second (10\% for $\mathrm{H} 1$ to $\mathrm{H} 2$ ) or third harmonic (5\% for $\mathrm{H} 2$ to $\mathrm{H} 3$ ) alone, in comparison with the first harmonic, and this with and without ACSA.

\subsection{Online communication}

Optimal parameters defined offline in group A were applied online to group B. For real-time communication, a PMTM feature extraction algorithm was used to retrieve the amplitude of the first harmonic at channels selected on the training set by ACSA. The mean offline training accuracy on all subjects of group B was $77.7 \pm 2.4 \%$. Results showed online accuracy greater than chance level for eight out of 12 healthy volunteers of group B (see figure 5). The mean online accuracy on all subjects of group B was $74.0 \pm 12.5 \%$. Considering only the eight subjects with an online accuracy higher than $70 \%$, the mean online accuracy was $80.3 \pm 8.6 \%$. In particular, control SC5B could answer correctly 32 questions out of 33 . The patients LIS5 and LIS6 preferred to stop the assessment after the training session due to fatigue. Offline accuracy of these patients were $70.5 \pm 3.9 \%$ and $58.0 \pm 5.5 \%$ respectively. Patient LIS3 answered successfully $70 \%$ of the questions. Patient LIS2 reached 64\%. Two patients (LIS1 and LIS4) achieved accuracy below chance level with a score of $52 \%$ (see figure 5).

\section{Discussion}

Eight out of the twelve healthy subjects succeeded in reaching a communication accuracy higher than the $70 \%$ CRR required for an efficient communication. In particular, one healthy subject achieved 32 out of 33 correct responses. Mean online accuracy on healthy subjects able to communicate was $80.3 \pm$ $8.6 \%$. Offline analysis showed that healthy subjects succeeded in reaching an average accuracy of $85 \%$ (with three subjects out of ten reaching more than $94 \%$ ), which exceeds accuracies of previous covert SSVEP-based BCIs [18-20]. Moreover, the proposed 'interlaced squares' stimulation pattern is small, portable, easy to use and adapted for bedside use with patients, features which are not shared by other covert stimulation devices requiring a cathode ray tube screen. Four out of the twenty-nine subjects involved in previous [24] and present studies showed performance at chance level. This illustrates that covert SSVEP-BCI systems may not be used by all subjects, as previously reported [34]. In group A, LAS and PMTM feature extraction algorithms obtained higher accuracies than classical Fourier transform, in accordance with previous observations [17]. CCA did not work when used in conjunction with ACSA. The achievement of this feature extraction algorithm depended on the input (all the channels together versus each channel separately), which could explain the decreased performance. We here suggest the use of an ACSA based on DSLVQ which leads to an $8 \%$ increase of accuracy.

While the use of higher harmonics has been shown to positively influence classification in overt SSVEP [16], adding the second and/or third harmonic did not improve the classification accuracy in our covert SSVEP paradigm. For healthy subjects and patients with LIS, the study of overt and covert power spectra illustrated the difference between the two conditions and the difficulties associated with covert SSVEP. We observed a decrease of the power at the target stimulation frequency, at the target's harmonic (at the level of intrinsic activity) and the presence of a peak at the non-target frequency with an amplitude close to the target amplitude (see figure 3). Therefore, adding harmonics did not add extra information.

In patients with LIS, two out of six obtained accuracies above chance level in the training session and one out of four was able to functionally communicate online. This low success rate can be partly explained by the clinical conditions of these patients: two patients stopped the test due to fatigue ; two other patients had a persistent nystagmus preventing effective perception of the stimuli. Concerning the ergonomics of the system, the patients LIS1, LIS2, LIS3 and LIS5 expressed that the system was easy to use and did not report visual or attentional problems (patients LIS4 and LIS6 expressed that the training part was too long). Future studies are needed to further assess the clinical pertinence of a fully independent system based on covert SSVEP in the LIS population. Our system should also be tested in a broader population of patients, including total LIS patients. The learning effect on communication performances, mental workload and user satisfaction should also be part of future researches. The accuracies obtained in patients are lower than those presented by (1) Parini et al [35] on patients with Duchenne muscular dystrophy using a four-class overt SSVEP-BCI and (2) Combaz et al [36] using an overt SSVEP speller in patients with incomplete LIS. Kübler and Birbaumer tested a visual P300 speller with patients [37]. Two out of five LIS patients were able to communicate with the system. However, all these systems were gaze-dependent, hence excluding patients without gaze control. This represents an important limitation for patients in which (1) a loss of gaze control is often observed in the chronic setting [38] and (2) eye movements in the acute setting may be inconsistent, very small and easily exhausted [15]. P300-based BCI have been studied to enable motorindependence. Kübler et al tested an auditory P300-speller in three patients with LIS [39]. While nine of the fourteen healthy subjects achieved spelling accuracy above $70 \%$, the patient's performance was poor. An auditory four-choice $\mathrm{P} 300$ speller BCI was proposed by Lulé $e t a l$ and was tested in two 


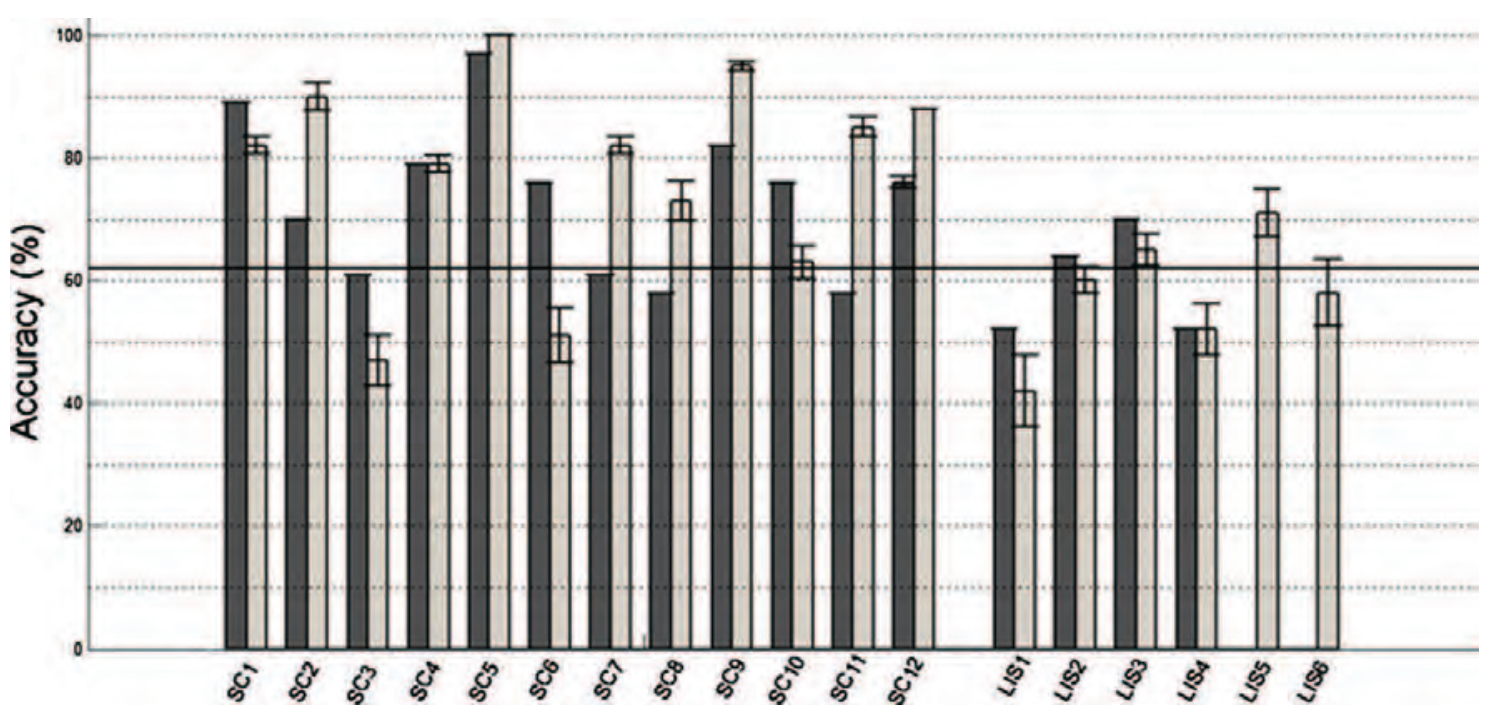

Figure 5. Recognition accuracy for healthy subjects (group B) and LIS patients. Dark gray represents online accuracy and light gray the, training accuracy. The horizontal full line represents the chance level for the training set.

patients with LIS [40]. One of the two patients showed an offline accuracy higher than chance level but neither of them showed an online performance higher than $70 \%$. Kübler and Birbaumer [37] also used slow cortical potential (SCP) and sensorimotor rhythms (SMR) as independent modalities. After a training period of a few months to years, three out of four classical LIS patients reached the $70 \%$ criterion level with SCP-BCI while none of the six complete LIS patients could communicate. Only one classical LIS and one complete LIS patient tried the SMR-BCI; the first reached a 77\% accuracy and the second remained at chance level. Limitations of these BCIs are the long user training required and the long sustained attention times needed for answering the questions. With a few seconds of focussed attention ( $7 \mathrm{~s}$ in our system) and none or little user training required, SSVEP-BCI could be a more adapted solution for clinical use than SMR and SCP-BCIs.

It is important to stress that SSVEP-based BCIs could evoke seizures. To avoid this potential risk, we here monitored EEG signals during each assessment in real-time. An EEG expert was present during all sessions to detect abnormal paroxystic electrical activity. We recorded no seizures in the present study. Using higher stimulation frequencies (i.e. 30$60 \mathrm{~Hz}$ ) are less epileptogenic but reduce SSVEP amplitudes [41].

\section{Conclusion}

We here demonstrated the feasibility of online communication with a covert SSVEP paradigm that is truly independent of neuromuscular function and gaze control [1]. We could functionally communicate with eight out of twelve healthy subjects. One out of four LIS patients could answer questions with the SSVEP-BCI system. The short sustained attention time $(7 \mathrm{~s})$, the concise training period and the robustness of our method could also make it a diagnostic tool for detecting command-following in severely brain-injured patients. Future studies should focus on improving patient accuracy and implementing an automated user-friendly online and asynchronous version of these novel BCI technologies.

\section{Acknowledgments}

The authors would like to thank all the patients for their collaboration. Steven Laureys is Research Director at the Fonds de la Recherche Scientifique (FRS). This work was supported by FEDER fund RADIOMED 930549, James McDonnell Foundation and French Speaking Community Concerted Research Action. The text reflects solely the views of its authors. The funders are not liable for any use that may be made of the information contained therein.

\section{References}

[1] Wolpaw J R, Birbaumer N, McFarland D J, Pfurtscheller G and Vaughan T M 2002 Brain-computer interfaces for communication and control Clin. Neurophysiol. $113767-91$

[2] Birbaumer N, Kübler A, Ghanayim N, Hinterberger T, Perelmouter J, Kaiser J, Iversen I, Kotchoubey B, Neumann N and Flor H 2000 The thought translation device (ttd) for completely paralyzed patients IEEE Trans. Neural Syst. Rehabil. Eng. 8 190-3

[3] Wolpaw J R, McFarland D J, Neat G W and Forneris C A 1991 An EEG-based brain-computer interface for cursor control Electroencephalogr. Clin. Neurophysiol. 78 252-9

[4] Farwell L A and Donchin E 1988 Talking off the top of your head: Toward a mental prosthesis utilizing event-related brain potentials Electroencephalogr. Clin. Neurophysiol. $70510-23$

[5] Cheng M, Gao X, Gao S and Xu D 2002 Design and implementation of a brain-computer interface with high transfer rates IEEE Trans. Biomed. Eng. 49 1181-6 
[6] Lalor E, Kelly S P, Finucane C, Burke R, Reilly R B and McDarby G 2004 Brain-computer interface based on the steady-state VEP for immersive gaming control Biomed. Tech. 49 63-64

[7] Middendorf M, McMillan G, Calhoun G and Jones K S 2000 Brain-computer interfaces based on the steady-state visual-evoked response IEEE Trans. Rehabil. Eng. 8 211-4

[8] Bin G, Gao X, Yan Z, Hong B and Gao S 2009 An online multi-channel ssvep-based brain-computer interface using a canonical correlation analysis method J. Neural Eng. 6 46002-8

[9] Perlstein W M, Cole M A, Larson M, Kelly K, Seignourel P and Keil A 2003 Steady-state visual evoked potentials reveal frontally-mediated working memory activity in humans Neurosci. Lett. 342 191-5

[10] Gray M, Kemp A H, Silberstein R B and Nathan P J 2003 Cortical neurophysiology of anticipatory anxiety: an investigation utilizing steady state probe topography (sspt) Neuroimage 20 975-86

[11] American Congress of Rehabilitation Medicine 1995 Recommendations for use of uniform nomenclature pertinent to patients with severe alterations of consciousness Arch. Phys. Med. Rehabil. 76 205-9

[12] Chatelle C, Chennu S, Noirhomme Q, Cruse D, Owen A and Laureys S 2012 Brain-computer interfacing in disorders of consciousness Brain Inj. 26 1510-22

[13] The Multi-Society Task Force on PVS 1994 Medical aspects of the persistent vegetative state New Engl. J. Med. 330 1499-508

[14] Majerus S, Gill-Thwaites H, Andrews K and Laureys S 2005 Behavioral evaluation of consciousness in severe brain damage Prog. Brain Res. 150 397-413

[15] Laureys S et al 2005 The locked-in syndrome: What is it like to be conscious but paralyzed and voiceless? Prog. Brain Res. 150 495-511

[16] Müller-Putz G R, Scherer R, Brauneis C and Pfurtscheller G 2005 Steady-state visual evoked potential (ssvep)-based communication : impact of harmonic frequency components J. Neural Eng. 2 123-30

[17] Müller-Putz G R, Eder E, Wriessnegger S C and Pfurtscheller G 2008 Comparison of dft and lock-in amplifier features and search for optimal electrode positions in ssvep-based bci J. Neurosci. Methods 168 174-81

[18] Kelly S P, Lalor E C, Reilly B and Foxe J J 2005 Visual spatial attention tracking using high-density ssvep data for independent brain-computer communication IEEE Trans. Neural Syst. Rehabil. Eng. 13 172-8

[19] Allison B Z, McFarland D J, Schalk G, Zheng S D, Jackson M M and Wolpaw J R 2008 Towards an independent brain-computer interface using steady state visual evoked potentials Clin. Neurophysiol. $119399-408$

[20] Zhang D, Maye A, Gao X, Hong B, Engel A K and Gao S 2010 An independent brain-computer interface using covert non-spatial visual selective attention J. Neural Eng. 7 16010-21

[21] Regan D 1989 Human Brain Electrophysiology: Evoked Potentials and Evoked Magnetic Fields in Science and Medicine (New York: Elsevier)

[22] Herrmann C S 2001 Eeg responses to 1-100 hz flicker: resonance phenomena in visual cortex and their potential correlation to cognitive phenomena Exp. Brain Res. $137346-53$

[23] Gao X, Xu D, Cheng M and Gao S 2003 A bci-based environmental controller for the motiondisabled IEEE Trans. Neural Syst. Rehabil. Eng. 11 137-40
[24] Lesenfants D, Partoune N, Soddu A, Lehembre R, Müller-Putz G R, Laureys S and Noirhomme Q 2011 Design of a covert ssvep-based BCI Proc. 5th Int. Brain-Computer Interface Conf. (Graz, Austria) pp 216-219

[25] Thomson D J 1982 Spectrum estimation and harmonic analysis Proc. IEEE 70 1055-96

[26] Hoogenboom N, Schoffelen J M, Oostenveld R, Parkes L M and Fries P 2006 Localizing human visual gamma-band activity in frequency, time and space Neuroimage $29764-73$

[27] Müller-Putz G R, Scherer R, Neuper C and Pfurtscheller G 2006 Steady-state somatosensory evoked potentials: Suitable brain signals for brain-computer interfaces? IEEE Trans. Neural Syst. Rehabil. Eng. 14 30-7

[28] Pregenzer M, Pfurtscheller G and Flotzinger D 1996 Automated feature selection with a distinction sensitive learning vector quantizer Neurocomputing 11 19-29

[29] Pregenzer M 1998 Dslvq-Distinct Sensitive Learning Vector Quantization (Aachen: Shaker Verlag)

[30] Nichols T E and Holmes A P 2002 Nonparametric permutation tests for functional neuroimaging: a primer with examples Hum. Brain Mapp. 15 1-25

[31] Schalk G, McFarland D J, Hinterberger T, Birbaumer N and Wolpaw J R 2004 Bci2000: a general-purpose brain-computer interface (bci) system IEEE Trans. Biomed. Eng. 51 1034-43

[32] Oostenveld R, Fries P, Maris E and Schoffelen J-M 2011 Fieldtrip: Open source software for advanced analysis of MEG, EEG, and invasive electrophysiological data Comput. Intell. Neurosci. 2011156869

[33] Müller-Putz G R, Scherer R, Brunner C, Leeb R and Pfurtscheller G 2008 Better than random? a closer look on bci results Int. J. Bioelectromagnetism 10 52-55

[34] Chen Y, Seth A K, Gally J A and Edelman G M 2003 The power of human brain magnetoencephalographic signals can be modulated up or down by changes in an attentive visual task Proc. Natl Acad. Sci. 6 3501-6

[35] Parini S, Maggi L, Turconi A C and Andreoni G 2009 A robust and self-paced BCI system based on a four class ssvep paradigm: algorithms and protocols for a high-transfer-rate direct brain communication Comput. Intell. Neurosci. 2009864564

[36] Combaz A, Chatelle C, Robben A, Vanhoof G, Goeleven A, Thijs V, Van Hulle M and Laureys S 2009 A comparison of two spelling brain-computer interfaces based on visual p3 and ssvep in locked-in syndrome Plos One 8 e 73691

[37] Küebler A and Birbaumer N 2008 Brain-computer interfaces and communication in paralysis: extinction of goal directed thinking in completely paralysed patients? Clin. Neurophysiol. 119 2658-66

[38] Alexandre M F, Challe G, Pradat-Dieh P and Le Hoang P 2012 Locked-in syndrome et vision : à propos de 13 cas Rev. Neurologique 168 A76

[39] Kübler A, Furdea A, Halder S, Hammer E M, Nijboer F and Kotchoubey B 2009 A brain-computer interface controlled auditory event-related potential (p300) spelling system for locked-in patients Ann. NY Acad. Sci. $115790-100$

[40] Lulé D et al 2013 Probing command following in patients with disorders of consciousness using a brain-computer interface Clin. Neurophysiol. 124 101-6

[41] Bakardjian H, Tanaka T and Cichockia A 2010 Optimization of ssvep brain responses with application to eight-command brain-computer interface Neurosci. Lett. 469 34-8 